\title{
Painless umbilical nodule in a nulliparous female - A clinical quandary
}

\author{
Aparna Muralidhar ${ }^{1}$, Nidha Gaffoor ${ }^{1}$ and Archana Shetty ${ }^{1,}$ \\ ${ }^{1}$ Department of Pathology, Dr. Chandramma Dayanada Sagar Institute of Medical education \& Research, Ramanagara, Karnataka-562112, \\ India
}

\begin{abstract}
Background: Endometriosis is a well-known entity most often found within the pelvis and extra pelvic sites like skin, diaphragm, gastrointestinal tract and brain. Primary umbilical endometriosis is a rare form of cutaneous endometriosis, typically presenting as a hyperpigmented umbilical nodule with or without cyclical pain. We present a case of painless umbilical nodule with discolouration in a nulliparous woman, unsuspected clinically with the diagnosis being made primarily on cytology.

Case report: A forty-year old female presented with a painless, brownish discoloured umbilical nodule, which was present since two months, was irreducible and had a negative cough impulse. She had no prior surgeries. Ultrasonography of abdomen and pelvis revealed a hypodense lesion of $2 \mathrm{~cm}$ in the umbilicus, suggesting a possibility of umbilical granuloma. Uterus showed leiomyomata. Cytological evaluation of the umbilical nodule was suggestive of endometriosis. The patient underwent hysterectomy for leiomyomata and omphalectomy. Histopathology confirmed the diagnosis of umbilical endometriosis supported by the immunohistochemistry marker (CD10) being positive for endometrial stromal cells. The uterus had adenomyotic foci in addition to leiomyomata.
\end{abstract}

Conclusion: The clinical distinction between primary umbilical endometriosis and other causes of umbilical nodules is challenging. Imaging modalities do not show pathognomonic signs in establishing this diagnosis. A definitive diagnosis is possible on cytology based on classical morphological features with histopathological examination being the gold standard diagnostic modality.

Keywords: endometriosis; endometrial stroma; cytology; immunohistochemistry; umbilicus; nulliparous

*Corresponding author: Dr. Archana Shetty, Associate Professor, Department of Pathology, Dr. Chandramma Dayananda Sagar Institute of Medical Education and Research (CDSIMER), Devarakaggalahalli post, Harohalli, Kanakapura Road, Ramanagara District Karnataka-562112, India. Mobile: +91 9986577343; Email: archanashetty2924@gmail.com

Received 12 April 2021; Revised 10 June 2021; Accepted 21 June 2021; Published 28 June 2021

Citation: Muralidhar A, Gaffoor N, Shetty A. Painless umbilical nodule in a nulliparous female - A clinical quandary. J Med Sci
Res. 2021; 9(3):169-172. DOI: http://dx.doi.org/10.17727/ JMSR.2021/9-24

Copyright: (C) 2021 Muralidhar A et al. Published by KIMS Foundation and Research Center. This is an open-access article distributed under the terms of the Creative Commons Attribution License, which permits unrestricted use, distribution, and reproduction in any medium, provided the original author and source are credited. 


\section{Introduction}

Endometriosis is defined by the presence of ectopic endometrial tissue at a site outside the uterus [1]. It is an oestrogen dependent condition, which was first described by Rokitansky in 1860 [2]. Common sites include ovaries, uterine ligaments, rectovaginal septum, Cul de sac, pelvic peritoneum, serosa of the bowel, appendix, mucosa of the cervix, vagina, fallopian tubes and laparotomy scars in decreasing order of frequency [1]. The skin is a relatively uncommon site.

Umbilical endometriosis, a form of cutaneous endometriosis, can be primary or secondary [3]. Primary umbilical endometriosis (PUE) is a rare subtype of spontaneous occurring cutaneous endometriosis estimated to account for only $0.5 \%$ to $1 \%$ of all extragenital endometriosis $[4,5]$. It is also known as Villar's nodule (with reference to the physician who first described it in 1886) [6]. Umbilical endometriosis is categorized as secondary when it occurs following open or laparoscopic procedures, the former being more common [7].

We present a clinically indubitable case of primary umbilical endometriosis with complete diagnostic workup, supported by insights from literature on this rare entity.

\section{Case report}

A forty year old unmarried, nulliparous woman presented to the surgery department of our hospital with a two month history of a gradually evolving painless swelling with discolouration over umbilicus. There was no history of abdominal trauma. Past medical/ surgical history and menstrual history were unremarkable. There was no history of any hormonal treatment in the past. The patient did not give any history of cyclical pain associated with the swelling. On physical examination, a firm, hyperpigmented, non tender nodule of around $2 \mathrm{~cm}$ involving the umbilicus was observed. The swelling was irreducible by gentle digital pressure. Cough impulse to rule out umbilical hernia was negative. Ultrasonography revealed a hypodense nodule of $2 \mathrm{~cm}$ at the umbilicus, favouring the possibility of umbilical granuloma (Figure 1). The uterus was bulky with thickened endometrium and a large posterior wall sub-serosal fibroid with calcifications. Bilateral ovaries were visualised and appeared normal. (a)

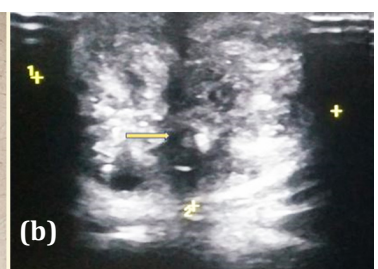

Figure 1: (a) Patient presenting with a hyper pigmented umbilical nodule; (b) Ultrasonography showing an illdefined hypoechoic nodule in umbilicus (arrow mark).

Fine needle aspiration of the umbilical nodule was performed. Smears were cellular comprising of epithelial and stromal fragments. The epithelial fragments were monolayered sheets of glandular cells with round to oval nuclei and moderate amount of cytoplasm, many in honeycomb pattern. Stromal cells were seen as fragments of spindle cells. Altered blood, debris and foamy macrophages some with ingested hemosiderin pigment were seen in the background (Figure 2). A diagnosis of umbilical endometriosis was conferred.

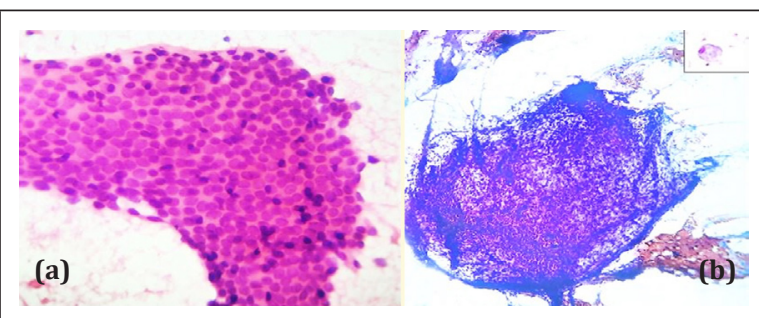

Figure 2: (a) Photomicrograph showing monolayered sheets of glandular epithelial cells, H\&E, 400x; (b) Photomicrograph showing fragments of stromal spindle cells, Pap stain, 100x. Inset - foamy macrophage, 400x.

The patient underwent omphalectomy for the umbilical nodule along with hysterectomy for fibroids. On gross examination the nodule measured $3.5 \times 3 \times 3.5 \mathrm{~cm}$. Cut section showed grey white areas with tiny haemorrhagic and cystic spots dispersed all over (Figure 3).

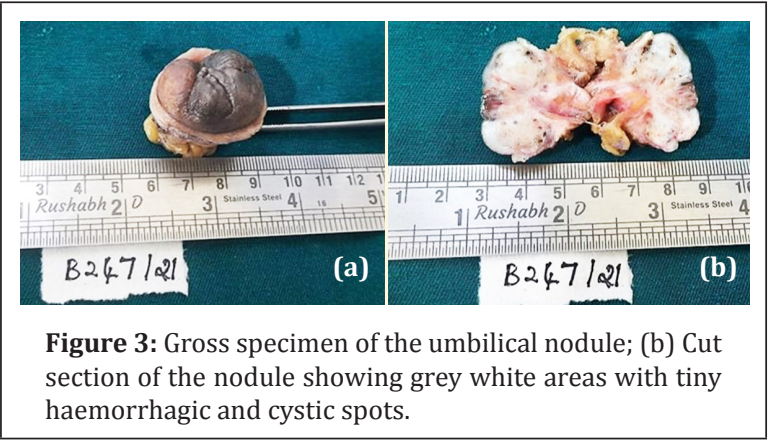


Microscopy of the umbilical nodule showed a lining of skin with underlying fibroadipose tissue. Subepithelially, foci of endometrial glands were seen surrounded by stroma. Immunohistochemistry with CD 10 showed immunoreactivity in the stromal cells (diffuse cytoplasmic and membranous staining), thereby supporting the diagnosis of umbilical endometriosis (Figure 4).

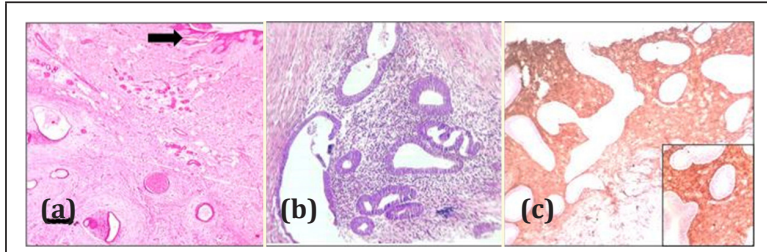

Figure 4: (a) Photomicrograph showing skin (arrow mark) with subepithelial endometrial glands and stroma, H\&E,400x; (b) Photomicrograph showing endometrial glands surrounded by stroma, H\&E,400x; (c) Photomicrograph showing CD10 positivity in stromal cells , 100x. Inset $-400 x$

Also seen were areas of haemorrhage and hemosiderin laden macrophages (Figure 5a). One of the blood vessels showed endometrial glands and stroma within the lumen (Figure 5b). Focal stromal myxoid change was noted. There were no features of endometrial hyperplasia, atypia or malignancy. The hysterectomy specimen revealed proliferative phase of endometrium and foci of adenomyosis in the myometrium along with leiomyomata.

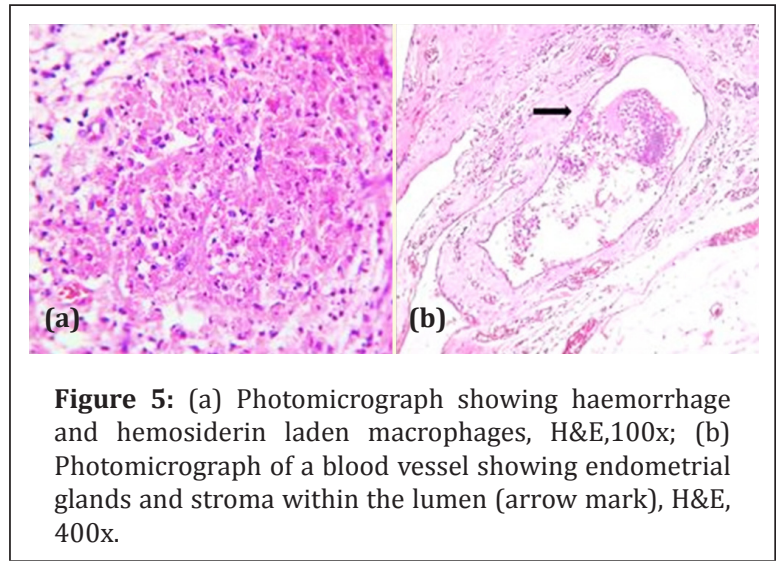

\section{Discussion}

Endometriosis is a benign disorder affecting $6-10 \%$ of all women in the reproductive age group [2]. Theories of aetiology include: regurgitation via retrograde flow of menstrual endometrium, hematogenous and lymphatic spread, implantation, coelomic metaplasia and differentiation form bone marrow progenitor cells [1]. Cutaneous endometriosis accounts for less than $1 \%$ of cases of ectopic endometrial tissue [8]. It is subdivided into primary and secondary depending on patients' surgical history.

Secondary cutaneous endometriosis is associated with prior abdominal or pelvic surgery. The prevailing pathogenesis is that endometrial cells dislodged during surgery seed the wound within and adjacent to the incision sites [3]. Primary cutaneous endometriosis has no relationship with surgical procedures. The former is more common, the most frequent site being umbilicus [9]. This form is postulated to occur as a consequence of endometrial cells migrating to the umbilicus via hematogenous or lymphatic routes and/ or originate from remnants of embryonic cells in the umbilical fold [7]. Another suggested theory is that umbilicus acts as a physiological scar with a predilection for ectopic endometrial implants [10]. One of the histologic sections of the lesion in our case showed a vascular channel with endometrial glands and stroma within (Figure 5b), supporting the theory of hematogenous spread.

Insights into the geneticrisk factors for endometriosis have revealed alterations in p53, PTEN, cytochrome P450 1A1, and Peroxisome proliferator-activated receptor $\gamma 2$ Pro-12- Ala. Prolonged radiation exposure and increased estrogen exposure have also been considered as contributing factors [2]. Literature reports $15-25 \%$ occurrence of concurrent pelvic endometriosis $[11,12]$.

There are very few reported cases of concurrent adenomyosis [13]. Our case had an incidental finding of adenomyosis as well. Both endometriosis and adenomyosis are known to share some key aspects: (i) common key molecular events in lesional development, i.e. epithelial mesenchymal transition, fibroblast-to-myofibroblast trans-differentiation, smooth muscle metaplasia and fibrogenesis, (ii) estrogen dependency, (iii) association with cyclical bleeds and (iv) common drugs for treatment.

However, recent literature [13] postulates that the respective lesional microenvironments, and hence the pathogenesis of both are not entirely the same. The patient in our case had concurrent adenomyosis, 
which makes us ponder that the co-existence of the two lesions may have common developmental factors, but different pathogenesis.

Survival of the ectopic endometrial tissue depends on local and systemic factors. Microvascular endothelial injury following implantation stimulates inflammation, which may enhance adhesion of tissue implants via production of adhesion molecules such as integrin and e-cadherins [9]. The endometrium in most lesions responds to the normal hormonal influences of the menstrual cycle [8]. The typical presentation is in the form of a bluish/purple mass, accompanied with catamenial bleeding from the umbilicus with or without associated pain or tenderness [5]. Our patient had no bleeding from the nodule nor cyclical pain associated with the nodule. Hence, a definitive diagnosis of umbilical endometriosis could not be established clinically and radiologically. FNAC can be supplementary, but inconclusive results have been reported in up to $75 \%$ cases [7]. However, in our case, FNAC was decisive, as it clinched the diagnosis based on classic cytological features.

Ultrasound is useful in defining whether a mass is cystic or solid, but it has low specificity for diagnosing endometriosis. It helps determine the anatomic relationship to the surrounding vital structures, thereby guiding the surgeon during the operative procedure if any. The other differential diagnoses to be considered radiologically are neoplasms (like sarcoma or lymphoma), umbilical granuloma, ventral hernia, abscess or haematoma [14].

Histopathological examination is the current gold standard for diagnosing PUE. In cases of suspected endometriosis, especially in the extra pelvic sites, where the reporting pathologist is unsure whether or notendometrial-typestromaispresent,CD10staining can be a good adjunct in identifying endometrial stroma [15]. Malignant transformation occurs in less than $1 \%$ of the cases, the most common being endometrioid type followed by clear cell carcinoma [15]. Surgical management is the treatment of choice $[4,12]$. Hormone therapy gonadotropin-releasing hormone agonists, oral contraceptive and danazol can be used preoperatively for relief of symptoms, but it is not curative $[9,12]$.

\section{Conclusion}

Cutaneous umbilical endometriosis is a rare entity, especially when it occurs spontaneously and needs to be considered in the differential diagnosis of umbilical nodules in females. Cytologic evaluation can play a pivotal role in clinically obscure cases, thereby aiding timely recognition and further management.

\section{Acknowledgement}

We thank the faculty from Departments of Surgery and $\mathrm{OBG}$ of our hopsital for providing their support.

\section{Conflicts of interest}

Authors declare no conflicts of interest.

\section{References}

[1] Ellenson LH, Pirog EC. The female genital tract. In: Kumar V, Abbas AK, Aster JC. Robbins and cotran pathologic basis of disease. 9th ed. Philadelphia: Elsevier Saunders; 2015; 1010-11.

[2] Giudice LC, Kao LC. Endometriosis. Lancet. 2004; 364 (9447):1789-1799.

[3] Raffi L, Suresh R, McCalmont TH, Twigg AR. Cutaneous endometriosis. Int J Womens Dermatol. 2019; 5(5):384386.

[4] Boesgaard-Kjer D, Boesgaard-Kjer D, Kjer JJ. Primary umbilical endometriosis (PUE). Eur J Obstet Gynecol Reprod Biol. 2017; 209:44-45.

[5] Taniguchi F, Hirakawa E, Azuma Y, Uejima C, Ashida K, et al. Primary umbilical endometriosis: unusual and rare clinical presentation. Case reports in obstetrics and gynecology. 2016; 9302376.

[6] Pariza G, Mavrodin CI. Primary umbilical endometriosis (Villar's nodule) - case study, literature revision. Chirurgia. 2014; $109(4): 546-549$.

[7] Victory R, Diamond MP, Johns DA. Villar's nodule: a case report and systematic literature review of endometriosis externa of the umbilicus. J Minim Invasive Gynecol. 2007; $14(1): 23-32$.

[8] Hurt MA, Weedon D. Weedon D. Weedon's Skin Pathology, 3rd ed. Philadelphia: Churchill Livingstone Elsevier; 2010. Dermatol Pract Concept. 2012; 2(1):79-82.

[9] Loh SH, Lew BL, Sim WY. Primary Cutaneous Endometriosis of Umbilicus. Ann Dermatol. 2017; 29(5):621-625.

[10] Nouland DVD, Kaur M. Primary umbilical endometriosis: a case report. Facts Views Vis Obgyn. 2017; 9(2):115-119.

[11] Chikazawa K, Mitsushita J, Netsu S, Konno R. Surgical excision of umbilical endometriotic lesions with laparoscopic pelvic observation is the way to treat umbilical endometriosis. Asian J Endosc Surg. 2014; 7(4):320-322.

[12] Makena D, Obura T, Mutiso S, Oindi F.Umbilical endometriosis: a case series. J Med Case Reports. 2020; 14:142.

[13] Saito A, Koga K, Osuga Y, Harada M, Takemura Y, et al. Individualized management of umbilical endometriosis: a report of seven cases. J Obstet Gynaecol Res. 2014; 40(1):4045 .

[14] Machairiotis N, Stylianaki A, Dryllis G, Zarogoulidis P, Kouroutou P, et al. Extrapelvic endometriosis: a rare entity or an under diagnosed condition? Diagn Pathol. 2013; 8:194.

[15] Agarwal N, Subramanian A. Endometriosis - morphology, clinical presentations and molecular pathology. J Lab Physicians. 2010; 2(1):1-9. 